\title{
The Influence of Cylindrical Inclusions on the Stability of a Directionally Solidified Interface
}

\author{
Layachi Hadj* \\ The University of Alabama, Department of Mathematics, Tuscaloosa, Alabama 35487
}

(Dated: November 21, 2018)

It is well known that the presence of an inclusion in the melt near a solidifying front induces a local deformation in the latter provided that the melt's thermal conductance differs from that of the inclusion 1, 2, 3, 4]. This local interfacial deflection is caused by the modification of the thermal gradient in the melt near the particle. The long time evolution of this local disturbance of the interface has been investigated for the case of a spherical particle in a pure substance [5], and in a binary alloy [6]. It is discovered that, provided that the particle-interface distance falls below a critical value, the induced perturbation grows and destabilizes the whole solid-liquid interface. This newly uncovered morphological instability is manifested only for some combination of the physical and processing parameters, and its onset is attributed to the reversal of the thermal gradient in the liquid gap between the particle and the interface [6]. This instability, whose characteristic size is of the order of the particle's diameter, occurs at pulling speeds that are below the threshold for the onset of the Mullins-Sekerka instability.

In reality, however, the inclusions have approximately cylindrical shapes. For example, in their study involving a particulate metal matrix composite of magnesium base alloy that is reinforced with $\mathrm{SiC}$ particles, Essa et. al [7] state that the inclusions resemble cylinders whose longitudinal sections can be approximated by rectangles of sides $a$ and $b(a \neq b)$. The pupose of this communication is to extend the stability analysis that was carried out for a spherical inclusion in 5$]$ to the more realistic case of a cylindrical inclusion. We let the inclusion have the more general shape of an elliptic cylinder, i.e. the longitudinal section is an ellipse of semi-axes $a$ and $b$ as illustrated in figure 1. The effect of the aspect ratio $c=b / a$, which emerges in the analysis as an important factor, will be examined.

Consider a system in which an inclusion is immersed in a bath of pure melt that is undergoing directional solidification. We ignore the gravitational effects and assume that the inclusion is both solid, i.e. nondeformable, and insoluble in the melt. Let the inclusion's position be such that the distance from its center to the planar solid-liquid interface is $Z_{0}$, and its axis of symmetry is parallel to the $Y$-axis. The problem, being invariant with respect to

${ }^{*}$ Electronic address: Lhadji@bama.ua.edu

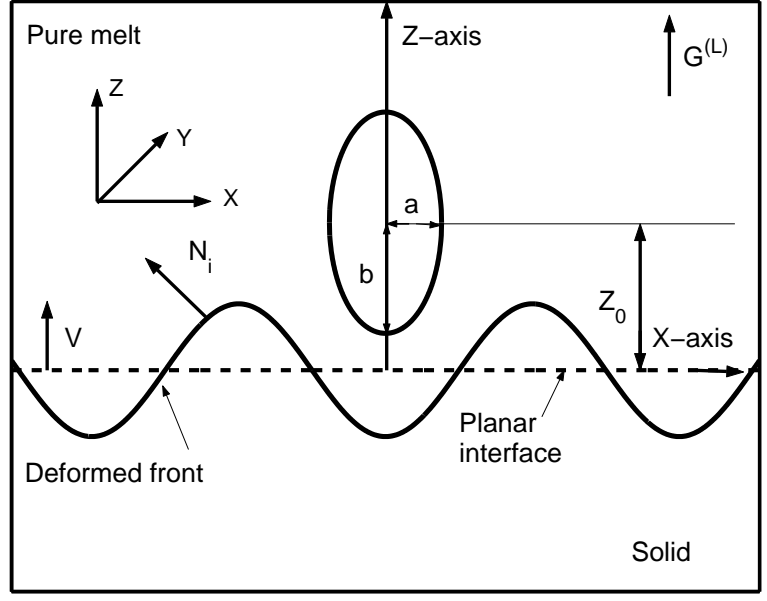

FIG. 1: A schematic diagram of a vertical solidification setup of a pure melt in which is embedded a cylindrical inclusion of aspect ratio $c=b / a$. The distance from the inclusion's center to the planar interface is $Z_{0}$. The cylinder's axis of symmetry is parallel to the $Y$-axis, and the planar interface coincides with the $X$-axis. The front's growth rate is $V$, $G^{(L)}$ is the imposed thermal gradient, and $\mathbf{N}_{i}$ is the normal to the interface pointing into the melt.

the $Y$-direction, is thus two-dimensional. The physical process is governed soleley by heat diffusion in the melt, solid, and inclusion with appropriate conditions at the interface, at the inclusion's surface, and at infinity. Let $T^{(q)}(X, Z)$ represent the temperature in phase $(q)$, where the superscript $(q)$ denotes symbolically the liquid phase, $(q=L)$, the solid phase, $(q=S)$, and inclusion, $(q=I)$, and the couple $(X, Z)$ represents the Cartesian coordinates of a point in the $X Z$-plane of a right-handed coordinate system relative to which the solid-liquid interface is at rest at $Z=0$, i.e. the $X$-axis. The melt occupies the top region, $Z>0$ as shown schematically in Fig. 1. The equation of the cylindrical inclusion, whose center is at the point $\left(0, Z_{0}\right)$, is given by

$$
\frac{X^{2}}{a^{2}}+\frac{\left(Z-Z_{0}\right)^{2}}{b^{2}}=1,
$$

where $a$ and $b$ are the semiaxes in the $X$ and $Y$ directions, respectively.. The nondimensionalization that is adopted here makes use of $a, a / V$, and the melting point $T_{m}$ as scales for length, time, and temperature. The following dimensionless system of equations and corresponding 
boundary conditions emerges,

$$
\begin{gathered}
\epsilon \lambda^{(q)}\left(\frac{\partial T^{(q)}}{\partial t}-\frac{\partial T^{(q)}}{\partial z}\right)=\Delta T^{(q)}, \\
T^{(S)}=T^{(L)}=1-\sigma \kappa, \quad \text { at } z=0, \\
\mathcal{S} v_{n}=k \frac{\partial T^{(S)}}{\partial n_{i}}-\frac{\partial T^{(L)}}{\partial n_{i}}, \quad \text { at } \quad z=0, \\
T^{(L)}=T^{(I)}, \quad \alpha \frac{\partial T^{(P)}}{\partial n_{P}}=\frac{\partial T^{(L)}}{\partial n_{P}}, \quad \text { on } \quad \partial I
\end{gathered}
$$$$
\frac{\partial T^{(S)}}{\partial z} \rightarrow G_{S}, \quad \frac{\partial T^{(L)}}{\partial z} \rightarrow G_{L}, \quad \text { as } \quad z \rightarrow-\infty \quad \text { and } \quad \infty .
$$

The symbols that appear in Eqs.(2)-(6) are defined in the following: $\Delta$ stands for the two-dimensional Laplacian, $\partial_{x x}+\partial_{z z}, \epsilon=a V / D^{(L)}$ is the Peclet number, and $\lambda^{(q)}=D^{(q)} / D^{(L)}$, where $D^{(q)}$ is the thermal diffusion coefficient in phase $(q)$. Equation (3) describes the continuity of temperature at the interface, with an interface equilibrium temperature that accounts for the Gibbs-Thomson effect. The parameter $\sigma=\sigma_{S L} / a L$ is the surface energy parameter, with $\sigma_{S L}$ being the interface excess free energy, $L$ is the latent heat of fusion per unit volume, and $\kappa$ is the front's curvature considered to be positive when the solid bulges into the melt. Equation (4) describes the heat balance at the interface, where $S=L V a /\left(T_{m} k^{(L)}\right)$ is the Stefan number, $v_{n}$ is the normal interface velocity, $k=k^{(S)} / k^{(L)}$, and $\partial / \partial n_{i}$ is the normal derivative at the solid-liquid interface. Equation (5) represents the continuity of temperature and of the heat flux on the inclusion's boundary $\partial I$, where $\alpha=k^{(P)} / k^{(L)}$ is the thermal conductance ratio, and $\partial / \partial n_{P}$ is the normal derivative at the inclusion's surface. Finally, Eq. (6) describes the far field conditions for the temperature, where $G_{S}=a G^{(S)} / T_{m}$ and $G_{L}=a G^{(L)} / T_{m}$, with $G^{(L)}$ and $G^{(S)}$ being the externally imposed (dimensional) thermal gradients in the liquid and solid phase, respectively.

The average size of the reinforcements that are typically used in the manufacture of PMMCs is about $12 \mu \mathrm{m}$ 7], while the melt's thermal diffusion coefficient is of the order $10^{-5} \mathrm{~m}^{2} / \mathrm{s}[8]$. Thus, if we assume that the growth rate is low enough, say $V \approx 10^{-8} \mathrm{~m} / \mathrm{s}$, then the corresponding Peclet number $\epsilon \approx 10^{-7}$. Note that the smallness of $\epsilon$ is due to the fact that it is proportional to the product of the velocity and the particle's radius, both of which are considered very small. Thus, the steady state form of Eq. (2) satisfies

$$
\Delta T^{(q)}+\epsilon \lambda^{(q)} \frac{\partial T^{(q)}}{\partial z}=\Delta T^{(q)}+O(\epsilon) .
$$

As in ref. [6], we could take advantage of the smallnes of $\epsilon$ and consider the expansions, with $\epsilon \ll 1$, $T^{(q)}=T_{B}^{(q)}+\epsilon \theta^{(q)}+O\left(\epsilon^{2}\right)$ and, for the interface, $z=0+\epsilon \eta+O\left(\epsilon^{2}\right)$. At order unity, the base state satisfies Laplace's equations with coupled boundary conditions at the inclusion's surface and at the planar interface. This problem is mathematically tractable, and the next order perturbations are easily calculated. We refer the interested reader to [6] for details. However, for both simplicity in the calculations and clarity in the presentation, we set $\epsilon=0$ in the present work. This simplification will not alter the key qualitative features of the instability mechanism that is the subject of this communication.

It is known that the thermal field in an infinite medium in which is embedded an inclusion of different material is given by $[9]$,

$$
T_{B}^{(L)}(x, z)=1+G_{L} z+\frac{(1-\alpha) G_{L}}{1+\mathcal{C}_{0}(\alpha-1)}\left(z+n H_{0}\right) \mathcal{C}_{\lambda(x, z)},
$$

where

$$
\mathcal{C}_{\lambda(x, z, n)}=\frac{c}{2} \int_{\lambda(x, z, n)}^{\infty} \frac{d u}{\left(c^{2}+u\right)^{3 / 2}(1+u)^{1 / 2}},
$$

$c=b / a, H_{0}=Z_{0} / a$, and $\lambda(x, z, n)$ is the positive root of the equation,

$$
\frac{x^{2}}{1+\lambda(x, z, n)}+\frac{\left(z-n H_{0}\right)^{2}}{c^{2}+\lambda(x, z, n)}=1 .
$$

For an elliptic cylinder, Eq. (9) reduces to

$$
\mathcal{C}_{\lambda(x, z, n)}=\frac{c}{1-c^{2}}\left[\sqrt{\frac{1+\lambda(x, z, n)}{c^{2}+\lambda(x, z, n)}}-1\right],
$$

while for a circular cylinder, we have

$$
\begin{gathered}
\mathcal{C}_{\lambda(x, z, n)}=\frac{1}{2\left[x^{2}+\left(z-n H_{0}\right)^{2}\right]}, \quad \text { and } \\
\lambda(x, z, n)=x^{2}+\left(z-n H_{0}\right)^{2}-1 .
\end{gathered}
$$

This solution to Laplace's equation, Eq. (8), does not, however, satisfy the boundary condition at the planar interface, Eq.(3), i.e. $T_{B}^{(L)} \neq 1$ at $z=0$. As shown in [4], the isotherm at $z=0$ is deformed if the thermal conductance ratio $\alpha \neq 1$. This, in turn, implies that the interface profile, which conforms to the isotherm at $z=0$, is also deformed. However, in order to conduct a linear stability analysis of the planar interface, we must seek solutions to Eq. (7), with $\epsilon=0$, and corresponding boundary conditions, Eqs.(3)-(6), that admit a planar interface profile. This is achieved by making use of the method of images. We refer the interested reader to ref. 10] and references therein for more details on this method. We 


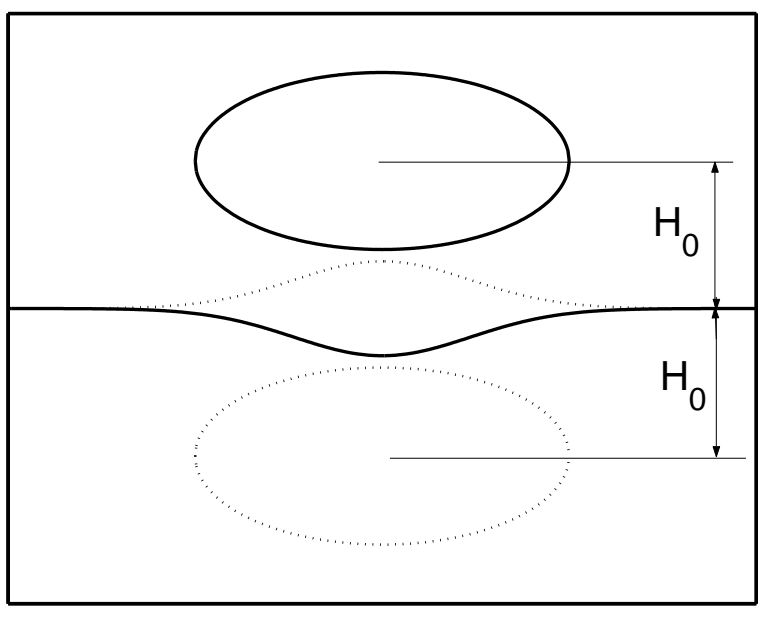

FIG. 2: A sketch of a highly heat conducting inclusion in the melt (continuous line) and its image (dotted line), and the associated deformed solid-liquid interface profile.

start by introducing an image inclusion that is centered at $\left(0,-H_{0}\right)$. On using the principle of superposition, the equation

$$
\begin{gathered}
T_{B}^{(L)}(x, z)=1+G_{L} z+\frac{(1-\alpha) G_{L}}{1+\mathcal{C}_{0}(\alpha-1)}\left[\left(z+H_{0}\right) \mathcal{C}_{\lambda(x, z)}+\right. \\
\left.\left(z-H_{0}\right) \mathcal{C}_{\lambda(x, z)}\right]
\end{gathered}
$$

not only satisfies Laplace's equation, but also the boundary condition, $T_{B}^{(L)}=1$ at $z=0$. This situation is depicted schematically in figure 2 for the case of an inclusion and its image that are both characterized by a thermal conductance ratio $\alpha>1$. According to [1, 2, 3, 4], the inclusion at $\left(0, H_{0}\right)$ induces a depression in the interface shape while its image at $\left(0,-H_{0}\right)$ induces a bump. The depression and the bump, being symmetrical about the $x$-axis, cancel out leaving the interface planar. Note, however, that Eq. (13) fails to satisfy the boundary condition at the inclusion's surface $\partial I$, i.e. Eq.(5). Thus, It is necessary to place another fictitious particle at $\left(0,2 H_{0}\right)$ and add its contribution to the thermal field. The resulting equation will then satisfy Eq. (5) but fails to satisfy Eq. (3). So an image inclusion is placed at $\left(0,-2 H_{0}\right)$ and so on and so forth. This process is continued indefinitely to yield,

$$
\begin{gathered}
T_{B}^{(L)}(x, z)=1+G_{L} z+\frac{(1-\alpha) G_{L}}{1+\mathcal{C}_{0}(\alpha-1)} \\
\quad \times \sum_{\substack{n=-\infty \\
n \neq 0}}^{\infty}\left(z+n H_{0}\right) \mathcal{C}_{\lambda(x, z, n)} .
\end{gathered}
$$

The expression, Eq.(14), now satisfies Laplace's equation and both boundary conditions, Eq.(3), at the planar in- terface, and Eq.(5) on $\partial I$. The thermal fields in the inclusion and the solid phase are described by

$$
\begin{gathered}
T_{B}^{(P)}(z)=\frac{G_{L}\left(z-H_{0}\right)}{1+\mathcal{C}_{0}(\alpha-1)}, \\
T_{B}^{(S)}(x, z)=1+G_{S} z+\frac{(1-\alpha) G_{L}}{k\left[1+\mathcal{C}_{0}(\alpha-1)\right]} \\
\times \sum_{\substack{n=-\infty \\
n \neq 0}}^{\infty}\left(z+n H_{0}\right) \mathcal{C}_{\lambda(x, z, n)} .
\end{gathered}
$$

Note that $T_{B}^{(S)}$ satisfies Laplace's equation, and $T_{B}^{(S)}=1$ at the planar interface $z=0$. The last term in Eq. (16) is necessary in order for $T_{B}^{(S)}$ to satisfy the heat balance equation at the planar interface, i.e. the planar interface moves with constant velocity; $T_{B}^{(S)}$ does not need to satisfy the boundary condition, Eq. (5), on the particle's surface $\partial I$.

We now examine the linear stability of the planar state defined by Eqs. (14)-(16) and corresponding boundary conditions at the planar interface. We first superimpose two-dimensional, time-dependent infinitesimal disturbances $\theta^{(L)}, \theta^{(S)}$ and $\eta$ upon the basic state solutions $T_{B}^{(L)}, T_{B}^{(S)}$ and $z=0$, respectively in Eq. (7) (without the convection term) and boundary conditions, Eqs. (3)-(6). The resulting equations are then linearized with respect to the disturbances to yield the following,

$$
\begin{gathered}
\Delta \theta^{(q)}=0, \\
\theta^{(L)}=-G_{L} \eta+\sigma \frac{\partial^{2} \eta}{\partial x^{2}}-F(x) \eta, \quad \text { at } z=0, \\
\theta^{(S)}=-G_{S} \eta+\sigma \frac{\partial^{2} \eta}{\partial x^{2}}-\frac{F(x)}{k} \eta, \quad \text { at } z=0, \quad(18) \\
\frac{\partial \theta^{(L)}}{\partial z} \rightarrow 0, \quad \text { as } z \rightarrow \infty, \quad \frac{\partial \theta^{(S)}}{\partial z} \rightarrow 0, \quad \text { as } \quad z \rightarrow-\infty \\
\frac{\partial \eta}{\partial t}=k \frac{\partial \theta^{(S)}}{\partial z}-\frac{\partial \theta^{(L)}}{\partial z}, \quad \text { at } z=0 .
\end{gathered}
$$

The small slope approximation in the expression for the curvature, i.e., $\kappa \approx-\partial^{2} \eta / \partial x^{2}$ has been used in Eqs.(18)(19), and

$$
F(x)=\frac{(1-\alpha) G_{L}}{1+\mathcal{C}_{0}(\alpha-1)} \sum_{\substack{n=-\infty \\ n \neq 0}}^{\infty}\left[\left(n H_{0}\right) \frac{\partial \mathcal{C}_{\lambda(x, n)}}{\partial z}+\mathcal{C}_{\lambda(x, n)}\right]
$$


The stability problem, Eqs. (17)-(21), is solved using the Fourier transform. We let $\mathcal{T}\{f(x, z, t)\}=\hat{f}(\omega, z, t)$. The transformed problem is

$$
\frac{\partial^{2} \hat{\theta}^{(q)}}{\partial z^{2}}-\omega^{2} \hat{\theta}^{(q)}=0
$$

with the accompanying conditions

$$
\begin{aligned}
& \hat{\theta}^{(L)}=-\left(G_{L}+\sigma \omega^{2}\right) \hat{\eta}-\mathcal{T}\{F(x) \eta\}, \quad \text { at } \quad z=0, \\
& \hat{\theta}^{(S)}=-\left(G_{S}+\sigma \omega^{2}\right) \hat{\eta}-\frac{\mathcal{T}\{F(x) \eta\}}{k}, \quad \text { at } \quad z=0,
\end{aligned}
$$

and with vanishing temperature gradients far away from the interface for $\hat{\theta}^{(L)}$ and $\hat{\theta}^{(S)}$. In order to make progress, we consider the approximation $\mathcal{T}\{F(x) \eta\} \approx F(0) \hat{\eta}$ since the major contribution to $F(x)$ comes from near the origin (see [5] for a similar approximation for the case of a spherical particle), where $F(0)$ can be be approximated by the leading asymptotic term for $c \approx 1$ (small deviation from a circular cylinder) as follows,

$$
F(0)=\frac{(\alpha-1) G_{L} c}{2\left[1+\mathcal{C}_{0}(\alpha-1)\right] H_{0}^{2}} \sum_{\substack{n=-\infty \\ n \neq 0}}^{\infty} \frac{1}{n^{2}}+O(c-1) .
$$

The approximation is, however, exact for a circular cylinder $(c=1)$. On using the fact that $\sum_{n=1}^{\infty} 1 / n^{2}=\pi^{2} / 6$ and $\mathcal{C}_{0}=1 /(c+1)$, we obtain

$$
F(0) \approx \frac{\pi^{2}(\alpha-1) G_{L} c(1+c)}{12(c+\alpha) H_{0}^{2}} .
$$

The solution of the transformed problem yields,

$$
\begin{gathered}
\hat{\theta}^{(L)}=-\left[G_{L}+\sigma \omega^{2}+F(0)\right] e^{-\omega z}, \\
\hat{\theta}^{(S)}=-\left[G_{S}+\sigma \omega^{2}+\frac{F(0)}{k}\right] e^{\omega z} .
\end{gathered}
$$

On imposing the heat balance equation at the interface, we obtain the following evolution equation for the interface perturbation,

$$
\frac{\partial \hat{\eta}}{\partial t}=\Omega(\omega) \hat{\eta}
$$

where $\Omega(\omega)=-\left[\mathcal{G}+(1+k) \sigma \omega^{2}+2 F(0)\right] \omega, \mathcal{G}=k G_{S}+G_{L}$ is the conductivity weighted thermal gradient, and $\Omega$ represents the growth rate of the interfacial perturbation. If $\Omega(\omega)<0$ then any initial disturbance dies out after a long time, and the planar interface is stable. If $\Omega(\omega)>0$ then the opposite scenario holds, and the interface is unstable. The marginal stability criterion is obtained by setting $\Omega(\omega)=0$. A plot of the growth rate as a function of the wavenumber $\omega$ is shown in figure 3 as function of the aspect ratio, $c$, and the inclusion-interface
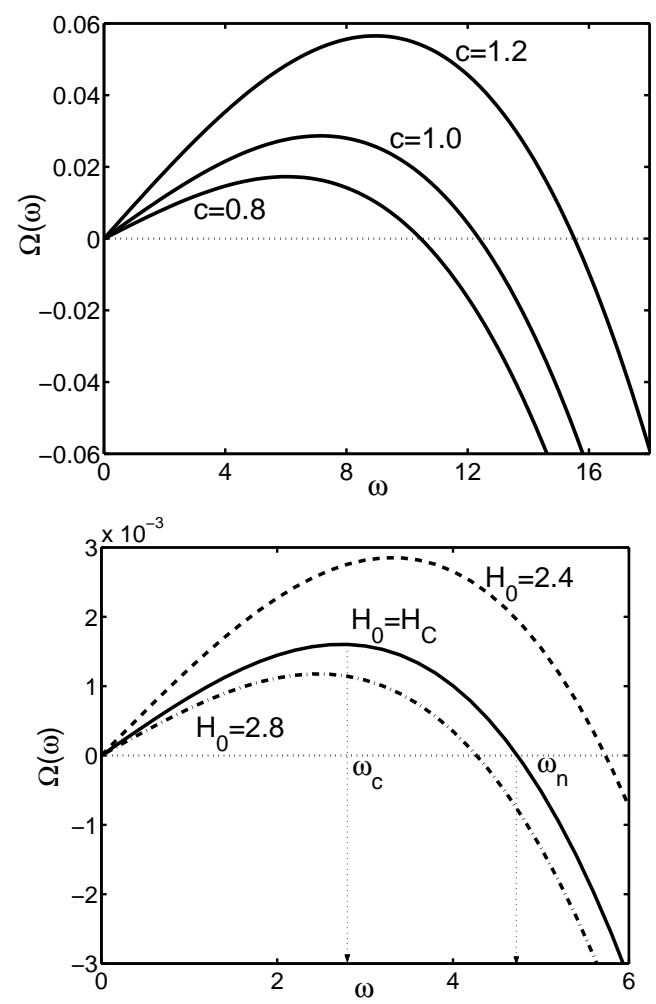

FIG. 3: A plot of the growth factor $\Omega$ versus the wavenumber $\omega$ for three values of the aspect ratio $c$ (plot on left), and for three values of the inclusion-interface distance $H_{0}$ (plot on right); $\omega_{c}$ is the critical wavenumber, Eq. (31), and $\omega_{n}$ is the neutral mode, i.e. $\Omega\left(\omega_{n}\right)=0$. The critical distance, $H_{c}$, is represented by a continuous line on the plot on the right.

distance, $H_{0}$. Note that the range of unstable wavenumbers, $0<\omega<\omega_{n}$, increases with the aspect ratio $c$, where $\omega_{n}$ is the neutral mode, i.e. $\Omega\left(\omega_{n}\right)=0$. Therefore, inclusions with larger $c$ values have a more destabilizing effect, or equivalently, the instability is onset at a larger inclusion-interface distance. The maximum growth rate occurs at the critical wavenumber $\omega_{c}$ that is obtained by maximizing $\Omega(\omega)$ with respect to $\omega$. We find,

$$
\omega_{c}^{2}=-\frac{2 F(0)+\mathcal{G}}{3(1+k) \sigma}
$$

with the understanding that the parameter values are such that the expression in Eq. (31) is positive, i.e. $F(0)<0$ and $(2 F(0)+\mathcal{G})<0$. On substituting Eq. (31) into the expression for the growth rate, $\Omega(\omega)$, we obtain the stability condition, $\mathcal{G}<-2 F(0)$, and on using Equation (27), we find the following stability criterion for an elliptic cylinder, valid for $c \approx 1$,

$$
H_{C} \approx \sqrt{\frac{\pi^{2} c(1+c) G_{L}(1-\alpha)}{6(c+\alpha) \mathcal{G}}},
$$

which reduces to the following exact criterion for a cir- 


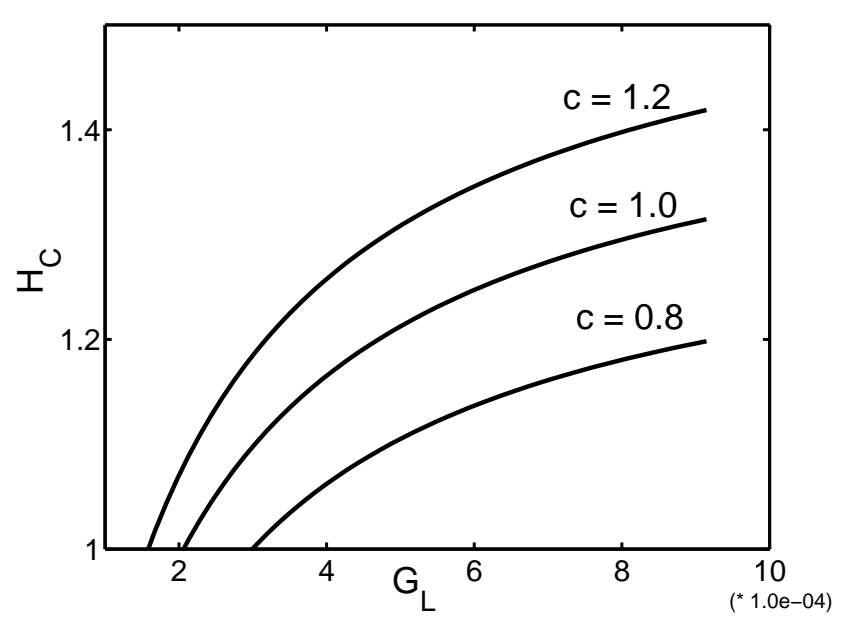

FIG. 4: Plot of the critical inclusion-interface distance, $H_{C}$ (Eq. (32)), as function of the thermal gradient $G_{L}$ (note the scale factor $1.0 e-04)$ for three distinct values of the aspect ratio $c, c=1.2, c=1.0$ and $c=0.8$, for a system consisting of succinonitrile $(\mathrm{SCN})$ containing an inclusion with $(\alpha=5)$. The regions of instability in the $H_{0}-G_{L}$ plane are below the curves. The numerical values of the parameters used in the calculations are taken from Ref. [1]. cular cylinder,

$$
H_{C}=\sqrt{\frac{\pi^{2} G_{L}(1-\alpha)}{3(1+\alpha) \mathcal{G}}} .
$$

The critical distance $H_{C}$ is the largest value of $H_{0}$ for the instability to appear. Note that the stability conditions, Eqs. (32)-(33), require that (i) the product $G_{L}(1-\alpha)>0$, and (ii) $H_{C}>1$. Therefore, the instability is observable only for certain combinations of the physical and processing parameters. Figure 4 depicts the plot of $H_{C}$ versus the dimensionless thermal gradient in the liquid for three distinct values of $c$. The numerical values of the parameters used in calculating $H_{C}$ pertain to an experimental system consisting of SCN containing an inclusion whose thermal conductance ratio, $\alpha$, is arbitrarily set equal to 0.5 . We note the following: (i) $H_{C}$ increases with the aspect ratio $c$, (ii) $H_{C}$ increases with $G_{L}$, and (iii) for every value of the aspect ratio $c$, there exists a lower bound for $G_{L}$ below which the instability is not observable, i.e. $H_{C}<1$. The dependence of $H_{C}$ on the inclusions size, i.e. $a$, and on other factors, such as $\alpha$, resemble that of the case of a spherical particle, with the exception that for spherical inclusions, $H_{C} \sim\left(G_{L} / \mathcal{G}\right)^{1 / 3}$. We refer the interested reader to [6] for more details.
[1] A.M. Zubko, V.G. Lobanov and V.V. Nikonova, Sov. Phys. Crystallgr. 18, 239 (1973).

[2] A.A. Chernov, D.E. Temkin and A.M. Mel'kinova, Sov. Phys. Crystallogr. 22 656-658 (1977).

[3] S. Sen, W.F. Faukler, P. Curreri and D.M. Stefanescu, Met. Trans. A 28, 2129 (1997).

[4] L. Hadji, Phys. Rev. E 64, 051502 (2001).

[5] L. Hadji, Scripta Materialia 48, 665 (2003).

[6] L. Hadji, Eur. Phys. J. B 37, 85-89 (2004).

[7] Y. E. Essa, J. Fernández-Sáez and J.L. PéresCastellanos, Composites Part B 34, 351 (2003).
[8] W. Kurz and D.J. Fisher, Fundamentals of Solidification, third edition (Trans. Tech. Publications, Switzerland, 1992).

[9] H.S. Carslaw and J.C. Jaeger, Conduction of Heat in Solids, second edition (Clarendon Press, Oxford, 1959).

[10] H. Cheng and L. Greengard, SIAM J. Appl. Math 58, 122-141 (1998).

[11] D. M. Stefanescu, R.V. Phalnikar, H. Pang, S. Ahuja, and B. K. Dhindaw, SIJ Int. 35, 300 (1995). 